Vol. 9, Issue 2, Dec 2019, 1-6

(C) TJPRC Pvt. Ltd.

\title{
DIGITAL VOTING SYSTEM USING BLOCKCHAIN TECHNOLOGY
}

\author{
SNEHAL BHOGAN ${ }^{1}$, BASIL JOSE ${ }^{1}$, GAURAV BHUSKUTE ${ }^{2}$, \\ VRUNDA GAWADE ${ }^{2}$, SUMEDHA SHIRODKAR ${ }^{2}$, \\ VENISHA GONSALVES ${ }^{2} \&$ VISHAL VARAK ${ }^{2}$ \\ ${ }^{I}$ Professor, Department of Computer Engineering,
} AITD, Goa University, India

${ }^{2}$ Student, Department of Computer Engineering, AITD, Goa University, India

\begin{abstract}
It's time to upgrade voting system using blockchain technology. Earlier people were able to trust each other upon a handshake but today it's extremely difficult to trust the digital world. It requires to authenticate identity and proving permission. With blockchain technology these two requirements are fulfilled. Blockchain is not an application nor a company. It is a solution for corruption. Democratic voting is critical and serious event in our country. By making use of blockchain which is a secure and robust system for digital voting can be devised. Traditional voting system uses centralized system, and there is one organization that manages it. It is possible to alter the database but by adopting blockchain technology which is a decentralized system we can prevent tampering and manipulation. Our proposed system achieves end-to-end verifiability using consensus algorithm.

We present our efforts to explore use of blockchain technology to seek solutions to problems in traditional voting system. Blockchain technology has positive impacts on our social life. This system will help increase the number of voters as well as the trust of people in their government.

KEYWORDS: Blockchain, Wallet, Cryptocurrency, Decentralized \& Bitcoin
\end{abstract}

Received: Apr 18, 2019; Accepted: May 08, 2019; Published: Jun 13, 2019; Paper Id.: IJCSEITRDEC20191

\section{INTRODUCTION}

Voting is a very important and serious event for any country that enables the general public to express their views by votes. Election process should be transparent and trustworthy. From using paper to vote to digital voting system the election process is changed to make it more secure.

A digital voting system uses an electronic device to cast vote. Although this system is cost efficient and fast it is still not that secure and not reliable. Recently United States proved that electronic machine can be hacked by hackers and alter the vote count easily. And the biggest problem is single virus can destroy the whole data storage. Fake display can be used and there is no way voter can validate their votes. One potential way of solving this problem is using blockchain technology.

Blockchain is a decentralized computation and information sharing platform. Blockchain is a decentralized database, it records all the transaction information in blocks. In simple words blockchain is a chain of blocks 
connected to each other. Blockchain technology mainly used in cryptocurrency bitcoin. Blockchain permit all the users to connect to the network, create blocks and verify the block. Each block has hash value that is valid till the block data is not altered. If the data is changed the hash also changes which may indicate malicious activity.

\section{LITERATURE SURVEY}

Paper [1] shows the proposed system consists of two blockchains, one stores voter's information and second stores for whom the voter has voted. Purpose of this project is to remove link of votes from certain parties and to keep track of vote record.

Blockchain stores information of the registered voters and allow them to vote on the election day. There is a threefactor authentication method which identifies the voter as the one who has registered. There is a double-check service method which ensures that voter's votes only once so that they are not forced to vote against their wish. There is an encryption mechanism which does not allow any person to hack. Private Key allows the user to read the vote present on the blockchain and decrypt the vote for election result.

In paper [2], Digital voting makes the electoral process cheaper, increases the speed of counting votes and makes the process simpler. This helps to establish democracy and gives the voter freedom to express their propositions. The blockchain system guarantees the voter security and privacy. Blockchain has overcome the manual voting system that is using pen and paper. Blockchain provides transparency for transferring thousands of votes per second.

Blockchain is totally depends on the smart contract which is written by the user. For larger cities some other actions has to be taken in consideration for example parent-child architecture which reduces the transactions stored. The system allows the voter to cast the vote from any location.

Paper [3], focuses on voting system was used to reduce errors and to increase the efficiency and to overcome the method of manual voting system. With the help of blockchain technology the number of steps were taken into consideration to explore the possibility of using blockchain. The proposed system provides advantages of blockchain that is cryptographic foundation and transparency. The system is focused on improving the confrontation of blockchain to "double spending problem" that is translated to "double voting" for electronic voting system. The system provides the benefits of checking the fake transaction and find an alternative solution to the problem. This system establishes end-to-end verifiable electronic voting.

\section{EXISTING SYSTEM}

The present voting system works manually. The system involves humans for counting the votes. In the present system the votes can be modified which may leads to miss-electing the candidate. The electronic voting machine is open to any malicious program and if it gets affected then any hacker can hack into the system

In election process even one vote is valuable. The system can get affected by a virus that can destroy the data storage. Fake displays can be installed which shows manipulated number, but fake votes are generated from the backend. Voters can not verify their vote, so it is easy for hackers to change a large number of votes. 


\section{PROPOSED SYSTEM}

Our system uses a block chain system which does not allow modification of data stored. We are using blockchain technology to secure our voting system by authenticating voters and saving the voter's count who took part in election. In blockchain there is no central body overseeing the result. This means that officials can confirm the voting results as soon as they are finished. With blockchain, each vote that is counted has to be completely and accurately verified.

\section{Architecture Diagram}

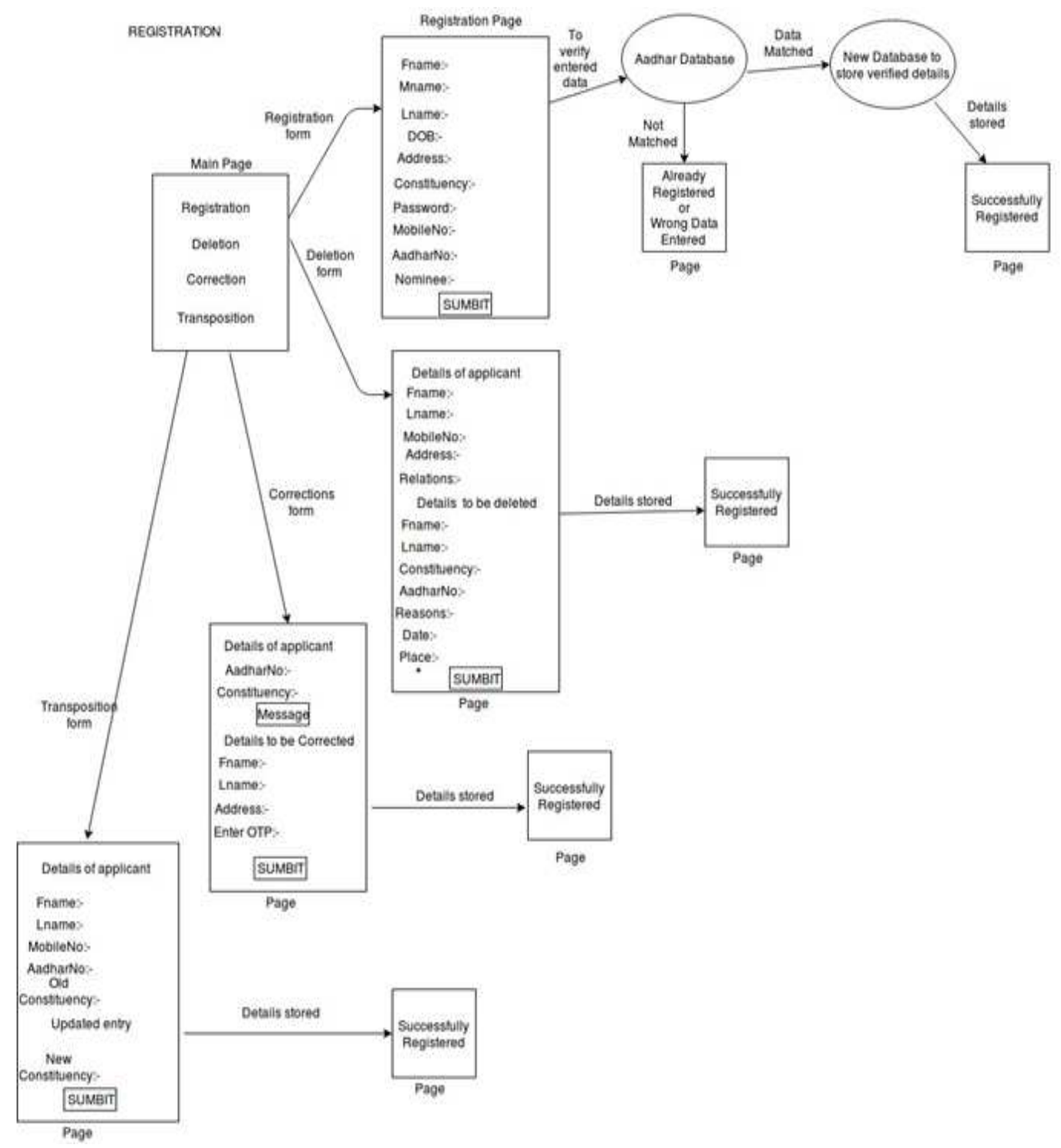

Figure 1: Registration Process 


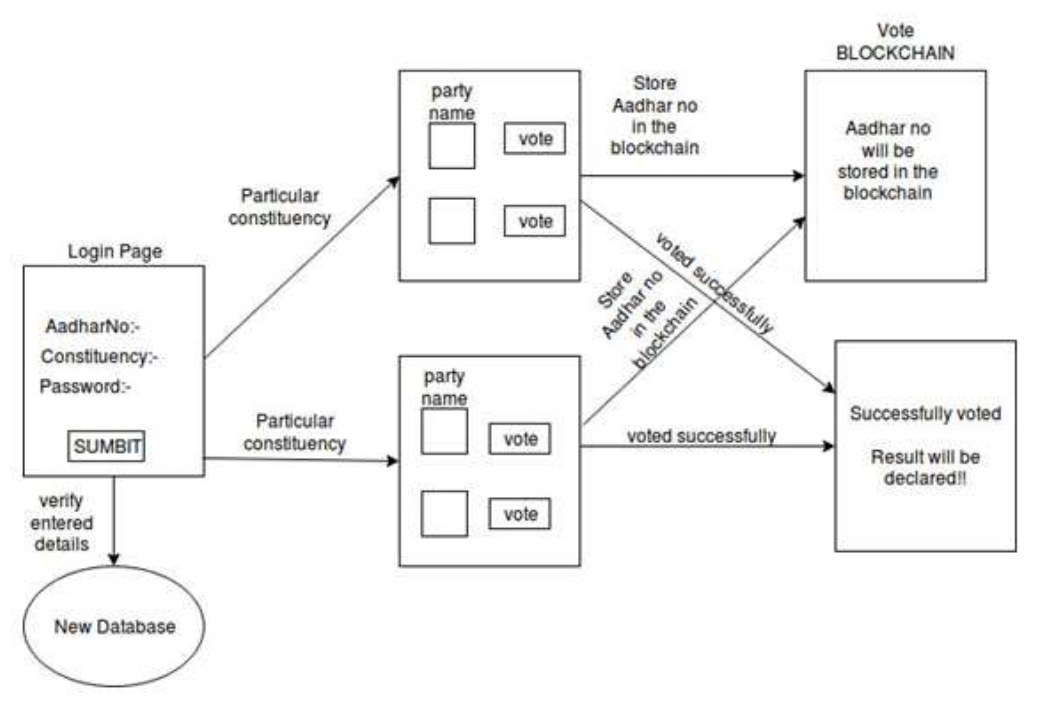

Figure 2: Voting Process

\section{PARTS OF THE SYSTEM}

There are three parts to the system. Each is explained below.

\section{User}

The user must have an Aadhar card which is used as verification ID to confirm that the voter is the citizen of the country. The user must first register himself in order to cast the vote during the elections for which he needs to have a laptop with a browser and a proper internet connection.

\section{Authentication}

The authentication is a traditional web server. The database connected to it stores the information of all the citizens in the country. This is used by voters to register to vote for their elections. People create login account when they register. It also checks the details entered by user i.e. first name, middle name, last name, DOB, address, constituency, password, mobile number, nominee name, nominee number and Aadhar. if the details match with Aadhar database then this are stored in the new database and a message is displaced saying "successfully registered". If the details don't match with the Aadhar database then a message is displayed saying "re-register" or "already registered" based on the type of error.

\section{Blockchain System}

The blockchain main aim is to not to allow modification in the data. The node then appends the transaction to the blockchain depending on the smart contracts that exist on each node. The smart contract is a protocol that the nodes follow not only to verify but also augment the vote in the system. Each node follows the smart contracts to verify and validate the vote. The blockchain is a protected system and is not accessible to anyone directly. The vote blockchain will store the Aadhar id in the hexadecimal form 


\section{PHASES IN THE SYSTEM}

The process is divided into two main parts the first part deals with the registrations and the second deals with the voting. The following are the important step to cast a vote. The other includes counting.

\section{Registering To Vote}

The registration process contains the registration form, deletion in the electoral roll and application for correction and transposition within the assembly. The process begins with the user interacting with the authentication via a website. The database linked contains the verified database by the government. The user enters the personal details which includes his/her first name, middle name, last name, DOB, address, constituency, password, mobile number, nominee name, nominee number and Aadhar. Once the user clicks on the submit button this details are checked with the database linked to it. Once the user has been verified his/her first name, middle name, last name, DOB, address, constituency, password, mobile number and Aadhar are stored in voters blockchain. Once this process has been done at the back end a pop up message appears for the user indicating successful registration along with the OTP and the date of elections being held on. If the registration fails due to any cause that might be failure in the user's internet connections or no data match then the user will be sent a pop up message to register. In the other case if the user is trying to register twice then a pop message will be displayed saying "you have been already registered!!"

In the deletion form nominee's full name, relation with the voter, mobile number, Aadhar id and his address is taken in order to keep record who has deleted the particular voter's details. The voters whose details are to be deleted his/her full name, constituency, Aadhar number, reason for deleting the data. The voter also has to enter the date when he has filled the form and place. Once the details matched and reason is verified automatically data will be deleted and will be notified to the nominee.

In the correction form voter who wants to correct his any details has to enter his Aadhar id and constituency when he/she clicks on the submit button a OTP will be sent to the number which he/she has entered during registrations. In the same form the user has to enter his full name, mobile number, address and the OTP and click on the submit button. Once he /she clicks on the submit button the particular changes will be reflected in the database. In the transposition form is to only change the constituency. The voter has to enter full name, old constituency, mobile number and Aadhar number and in the second part the user has to enter the new constituency which he/she is willing to change. Once he/she clicks on the submit button the details entered by the voter will be verified and changes will reflect in the database.

\section{Voting}

The voting process includes checking of the voters Id proof number on the admin system on the voting day to ensure that he/she is the real voter. The elections boot will have all the details of the voters.

Each election boot node will have a personal computer which will have the blockchain.

Once the voter has been verified by the admin the voter will be allowed to cast a vote. To do so the voter must enter his Id along with the password which he has entered during the registration time.

Once logged in successfully the user will be displayed with a page that will contain the candidates of the constituency which he/she has mentioned during registration. The page will display their name, photo, party which they 
belong and a symbol of representing the party with this there will be a button to cast the vote. Once the voter cast the vote, he/she has to click the submit button and log out and wait for the results to get display at the end of the elections.

\section{Counting of Votes}

When the voter cast the vote to its candidate the vote is immediately transferred in to the vote's blockchain i.e. in the voter blockchain there are nodes for all the locations each voter has its wallets assign value 1 . As soon as a voter casts a vote the token 1 from his/her wallet is sent to the candidate for whom he/she has voted.

However, this process continues of transacting of tokens from voter's wallet to candidates' wallets i.e. is from voter blockchain to the vote blockchain. At the end of the election the one candidate with the highest number of tokens in his wallet is announced to be the winner.

\section{CONCLUSIONS}

The traditional voting system has various issues. E-voting has replaced paper voting and increased efficiency and reduced errors but there a security issues such as tampering of votes, the machine can get affected by a virus and destroy data stored in the system and fake display can show manipulated votes and the tallying process is slow.

Our proposed system will solve all the issues faced in the voting process since it is reliable, tamper proof, efficient, easy to use and provides security.

We are focusing on improving the digital voting system by making use of Blockchain Technology by creating a public Blockchain which could be used for storing and counting votes.

This will not only build trust in government but also expose people to Blockchain Technology. Our system will use the user friendly web-based interface that will require minimum input from user

\section{REFERENCES}

1. Digital Voting with the use of Blockchain Technology, Andrew Barnes, Christopher Brake and Thomas Perry (https://www.economist.com/sites/default/files/plymouth.pdf)

2. Blockchain-Based E-Voting System, Friðrik P. Hjálmarsson, Gunnlaugur K. Hreiðarsson (https://skemman.is/bitstream/1946/31161/1/Research-Paper-BBEVS.pdf)

3. Secure Digital voting system based on blockchain technology Kashif Mehboob Khan, Junaid Arshad, Mohammad Mubashir Khan 